Bull. Korean Math. Soc. 49 (2012), No. 5, pp. 1027-1040

http://dx.doi.org/10.4134/BKMS.2012.49.5.1027

\title{
WEYL TYPE-THEOREMS FOR DIRECT SUMS
}

\author{
Mohammed Berkani AND Hassan Zariouh
}

\begin{abstract}
The aim of this paper is to study the Weyl type-theorems for the orthogonal direct sum $S \oplus T$, where $S$ and $T$ are bounded linear operators acting on a Banach space $X$. Among other results, we prove that if both $T$ and $S$ possesses property $(g b)$ and if $\Pi(T) \subset \sigma_{a}(S), \Pi(S) \subset$ $\sigma_{a}(T)$, then $S \oplus T$ possesses property $(g b)$ if and only if $\sigma_{S B F_{+}^{-}}(S \oplus T)=$ $\sigma_{S B F_{+}^{-}}(S) \cup \sigma_{S B F_{+}^{-}}(T)$. Moreover, we prove that if $T$ and $S$ both satisfies generalized Browder's theorem, then $S \oplus T$ satisfies generalized Browder's theorem if and only if $\sigma_{B W}(S \oplus T)=\sigma_{B W}(S) \cup \sigma_{B W}(T)$.
\end{abstract}

\section{Introduction}

Throughout this paper, let $L(X)$ denote the Banach algebra of all bounded linear operators acting on an infinite-dimensional complex Banach space $X$. For $T \in L(X)$, let $N(T), R(T), \sigma(T), \sigma_{a}(T)$, and $\sigma_{p}(T)$ denote respectively the null space, the range, the spectrum, the approximate point spectrum, and the point spectrum (i.e., the set of all eigenvalues) of $T$. We denote also by $\sigma_{p}^{0}(T)$ the set of all eigenvalues of $T$ of finite multiplicity, by $\rho(T)=\mathbb{C} \backslash \sigma(T)$ the usual resolvent set of $T$, and by $\rho_{a}(T)=\mathbb{C} \backslash \sigma_{a}(T)$ the approximate resolvent set of $T$. Let $\alpha(T)$ and $\beta(T)$ be the nullity and the deficiency of $T$ defined by $\alpha(T)=\operatorname{dim} N(T)$ and $\beta(T)=\operatorname{codim} R(T)$. Recall that an operator $T \in L(X)$ is called an upper semi-Fredholm operator if $\alpha(T)<\infty$ and $R(T)$ is closed, while $T \in L(X)$ is called a lower semi-Fredholm operator if $\beta(T)<\infty$. If $T \in L(X)$ is either an upper or a lower semi-Fredholm operator, then $T$ is called a semi-Fredholm operator, and the index of $T$ is defined by $\operatorname{ind}(T)=$ $\alpha(T)-\beta(T)$. If both $\alpha(T)$ and $\beta(T)$ are finite, then $T$ is called a Fredholm operator. An operator $T \in L(X)$ is called a Weyl operator if it is a Fredholm operator of index 0 . Let $S F_{+}(X)$ denote the class of all upper semi-Fredholm operators and define $S F_{+}^{-}(X)=\left\{T \in S F_{+}(X): \operatorname{ind}(T) \leq 0\right\}$. The Weyl essential approximate spectrum $\sigma_{S F_{+}^{-}}(T)$ of $T$ is defined by $\sigma_{S F_{+}^{-}}(T)=\{\lambda \in$

Received June 1, 2011.

2010 Mathematics Subject Classification. Primary 47A53, 47A10, 47A11.

Key words and phrases. property $(g b)$, property $(b)$, property $(g w)$, direct sums, essential semi-B-Fredholm spectrum.

The first author was supported by Protars D11/16 and PGR-UMP.

(C)2012 The Korean Mathematical Society 
$\left.\mathbb{C}: T-\lambda I \notin S F_{+}^{-}(X)\right\}$, the essential spectrum $\sigma_{e}(T)$ of $T$ is defined by $\sigma_{e}(T)=\{\lambda \in \mathbb{C}: T-\lambda I$ is not a Fredholm operator $\}$ and the Weyl spectrum $\sigma_{W}(T)$ of $T$ is defined by $\sigma_{W}(T)=\{\lambda \in \mathbb{C}: T-\lambda I$ is not a Weyl operator $\}$.

For $T \in L(X)$, let $\Delta(T)=\sigma(T) \backslash \sigma_{W}(T)$ and let $\Delta_{a}(T)=\sigma_{a}(T) \backslash \sigma_{S F_{+}^{-}}(T)$. Following Coburn [10], we say that Weyl's theorem holds for $T \in L(X)$ if $\Delta(T)=E^{0}(T)$, where $E^{0}(T)=\{\lambda \in \operatorname{iso} \sigma(T): 0<\alpha(T-\lambda I)<\infty\}$. Here and elsewhere in this paper, for $A \subset \mathbb{C}$, iso $A$ denotes the set of all isolated points of $A$.

According to Rakočević [21], an operator $T \in L(X)$ is said to satisfy a-Weyl's theorem if $\Delta_{a}(T)=E_{a}^{0}(T)$, where $E_{a}^{0}(T)=\left\{\lambda \in \operatorname{iso} \sigma_{a}(T): 0<\alpha(T-\lambda I)<\right.$ $\infty\}$. It is well known [21] that an operator satisfying a-Weyl's theorem satisfies Weyl's theorem, but not conversely.

Recall that the ascent $a(T)$, of an operator $T$, is defined by $a(T)=\inf \{n \in$ $\left.\mathbb{N}: c_{n}^{\prime}(T)=0\right\}$ where $c_{n}^{\prime}(T)=\operatorname{dim} \frac{N\left(T^{n+1}\right)}{N\left(T^{n}\right)}$ and the descent $\delta(T)$ of $T$, is defined by $\delta(T)=\inf \left\{n \in \mathbb{N}: c_{n}(T)=0\right\}$ where $c_{n}(T)=\operatorname{dim} \frac{R\left(T^{n}\right)}{R\left(T^{n+1}\right)}$, with $\inf \emptyset=\infty$. It is well known that $T \in L(X)$ is Drazin invertible if and only if it has a finite ascent and descent, which is also equivalent to the fact that $T=U \oplus V$, where $U$ is an invertible operator and $V$ is a nilpotent one (see [18, Corollary 2.2] and $\left[22\right.$, Proposition 6]). The Drazin spectrum $\sigma_{D}(T)$ of an operator $T$ is defined by $\sigma_{D}(T)=\{\lambda \in \mathbb{C}: T-\lambda I$ is not Drazin invertible $\}$. An operator $T \in L(X)$ is called Browder if it is a Fredholm of finite ascent and descent. The Browder spectrum $\sigma_{b}(T)$ of $T$ is defined by $\sigma_{b}(T)=\{\lambda \in \mathbb{C}: T-\lambda I$ is not Browder $\}$.

Let $\Pi(T)$ be the set of all poles of the resolvent of $T$ and let $\Pi^{0}(T)$ be the set of all poles of the resolvent of $T$ of finite rank, that is $\Pi^{0}(T)=\{\lambda \in \Pi(T)$ : $\alpha(T-\lambda I)<\infty\}$. According to [16], a complex number $\lambda$ is a pole of the resolvent of $T$ if and only if $0<\max (a(T-\lambda I), \delta(T-\lambda I))<\infty$. Moreover, if this is true, then $a(T-\lambda I)=\delta(T-\lambda I)$. According also to [16], the space $R\left((T-\lambda I)^{a(T-\lambda I)+1}\right)$ is closed for each $\lambda \in \Pi(T)$. An operator $T \in L(X)$ is said to satisfy Browder's theorem if $\Delta(T)=\Pi^{0}(T)$.

For $T \in L(X)$ and a nonnegative integer $n$, define $T_{[n]}$ to be the restriction of $T$ to $R\left(T^{n}\right)$ viewed as a map from $R\left(T^{n}\right)$ into $R\left(T^{n}\right)$ (in particular $T_{[0]}=T$ ). If for some integer $n$ the range space $R\left(T^{n}\right)$ is closed and $T_{[n]}$ is an upper (resp. a lower) semi-Fredholm operator, then $T$ is called an upper (resp. a lower) semi-B-Fredholm operator, see [7]. In this case and by [7, Proposition 2.1], $R\left(T^{m}\right)$ is closed, $T_{[m]}$ is a semi-Fredholm operator, and $\operatorname{ind}\left(T_{[m]}\right)=\operatorname{ind}\left(T_{[n]}\right)$ for each $m \geq n$. This enables us to define the index of the semi-B-Fredholm $T$ as the index of the semi-Fredholm operator $T_{[n]}$. Moreover, if $T_{[n]}$ is a Fredholm operator, then $T$ is called a B-Fredholm operator, see [3]. A semiB-Fredholm operator is an upper or a lower semi-B-Fredholm operator. An operator $T$ is said to be a B-Weyl operator if it is a B-Fredholm operator of index zero. The B-Weyl spectrum $\sigma_{B W}(T)$ of $T$ is defined by $\sigma_{B W}(T)=\{\lambda \in$ $\mathbb{C}: T-\lambda I$ is not a B-Weyl operator $\}$, and the B-Fredholm spectrum $\sigma_{B F}(T)$ of $T$ is defined by $\sigma_{B F}(T)=\{\lambda \in \mathbb{C}: T-\lambda I$ is not a B-Fredholm operator $\}$. 
For $T \in L(X)$, let $\Delta^{g}(T)=\sigma(T) \backslash \sigma_{B W}(T)$. According to [6], an operator $T \in L(X)$ is said to satisfy generalized Browder's theorem if $\Delta^{g}(T)=\Pi(T)$, and is said to satisfy generalized Weyl's theorem if $\Delta^{g}(T)=E(T)$, where $E(T)=\{\lambda \in \operatorname{iso} \sigma(T): \alpha(T-\lambda I)>0\}$. It is proved in [6] that an operator satisfying generalized Weyl's theorem satisfies Weyl's theorem, but the converse does not hold in general.

Let $S B F_{+}(X)$ be the class of all upper semi-B-Fredholm operators,

$$
S B F_{+}^{-}(X)=\left\{T \in S B F_{+}(X): \operatorname{ind}(T) \leq 0\right\} .
$$

The essential semi-B-Fredholm spectrum $\sigma_{S B F_{+}^{-}}(T)$ of $T$ is defined by

$$
\sigma_{S B F_{+}^{-}}(T)=\left\{\lambda \in \mathbb{C}: T-\lambda I \notin S B F_{+}^{-}(X)\right\} .
$$

Let $\Pi_{a}(T)$ be the set of all left poles of $T$ and $\Pi_{a}^{0}(T)$ be the set of all left poles of $T$ of finite multiplicity, that is $\Pi_{a}^{0}(T)=\left\{\lambda \in \Pi_{a}(T): \alpha(T-\lambda I)<\infty\right\}$. According to [6], a complex number $\lambda \in \sigma_{a}(T)$ is a left pole of $T$ if $a(T-$ $\lambda I)<\infty$ and $R\left((T-\lambda I)^{a(T-\lambda I)+1}\right)$ is closed. Hence we have always $\Pi(T) \subset$ $\Pi_{a}(T)$ and $\Pi^{0}(T) \subset \Pi_{a}^{0}(T)$. For $T \in L(X)$, let $\Delta_{a}^{g}(T)=\sigma_{a}(T) \backslash \sigma_{S B F_{+}^{-}}(T)$. According also to [6], an operator $T \in L(X)$ is said to satisfy generalized aBrowder's theorem if $\Delta_{a}^{g}(T)=\Pi_{a}(T)$ and is said to satisfy a-Browder's theorem if $\Delta_{a}(T)=\Pi_{a}^{0}(T)$. It is proved in [6] that if $T \in L(X)$ satisfies generalized a-Weyl's theorem $\Delta_{a}^{g}(T)=E_{a}(T)$, where $E_{a}(T)=\left\{\lambda \in\right.$ iso $\sigma_{a}(T): \alpha(T-\lambda I)>$ $0\}$, then it satisfies a-Weyl's theorem, but the converse is not true in general.

Definition 1.1. A bounded linear operator $T \in L(X)$ is called polaroid if all isolated points of the spectrum are poles of the resolvent of $T$, i.e., iso $\sigma(T)=$ $\Pi(T)$, and is called isoloid if all isolated points of the spectrum are eigenvalues of $T$, i.e., iso $\sigma(T)=E(T)$.

Definition 1.2 (See [17]). We will say that $T \in L(X)$ has the single valued extension property at $\lambda_{0}$, (SVEP for short) if for an arbitrary open neighborhood $\mathcal{U}$ of $\lambda_{0}, f=0$ is the only analytic function $f: \mathcal{U} \longrightarrow X$ such that $(T-\lambda I) f(\lambda)=0$ for all $\lambda \in \mathcal{U}$. We will say that $T$ has the SVEP if $T$ has this property at every point $\lambda \in \mathbb{C}$.

Following [1] and [20], we say that $T \in L(X)$ possesses property $(w)$ if $\Delta_{a}(T)=E^{0}(T)$. We say that $T \in L(X)$ possesses property $(g w)$ if $\Delta_{a}^{g}(T)=$ $E(T)$. The property $(g w)$ has been introduced and studied in [2], which is an extension to the context of B-Fredholm theory of the property $(w)$. It is shown $[2$, Theorem 2.3] that an operator possessing property $(g w)$ possesses property $(w)$, but not conversely.

Following [8, Definition 2.1], we say that $T$ possesses property $(b)$ if $\Delta_{a}(T)=$ $\Pi^{0}(T)$ and that $T$ possesses property $(g b)$ if $\Delta_{a}^{g}(T)=\Pi(T)$. It is proved in $[8$, Theorem 2.3] that an operator possessing property $(g b)$ possesses property $(b)$ but not conversely and it is proved in [8] that properties $(w)$ and $(g w)$ imply properties $(b)$ and $(g b)$ respectively, but the converses do not hold in general. 
Several authors $[12,19]$ considered Weyl's theorem for operator matrices, and in particular it is proved that Weyl's theorem is not transmitted from the direct summands $S$ and $T$ to the (orthogonal) direct sum $S \oplus T$. Nonetheless, they provided certain sufficient conditions on $S$ and $T$ which insures that Weyl's theorem lolds for $S \oplus T$. More recently, generalized Weyl's and generalized a-Weyl's theorem for orthogonal direct sums had been also examined in [13].

As it had been shown by S. V. Djordjević and Y. M. Han in [11], [14] and by R. E. Harte and W. Y. Lee in [15], the essential (resp. Browder) spectrum of a direct sum is the union of the essential (resp. Browder) spectra of its components and the Weyl (resp. Weyl essential approximate) spectrum of a direct sum is included in the union of the Weyl (resp. Weyl essential approximate) spectra of the summands. Then it is natural to ask if similar properties are valid in the B-Fredholm context. As we have expected the answer is yes and the results we obtain can be summarized as follows. So in the second section, we show that if $T$ and $S$ are bounded linear operators acting on a Banach space $X$, then $\sigma_{B F}(S \oplus T)=\sigma_{B F}(S) \cup \sigma_{B F}(T), \sigma_{D}(S \oplus T)=$ $\sigma_{D}(S) \cup \sigma_{D}(T), \sigma_{B W}(S \oplus T) \subseteq \sigma_{B W}(S) \cup \sigma_{B W}(T)$, and $\sigma_{S B F_{+}^{-}}(S \oplus T) \subseteq$

$\sigma_{S B F_{+}^{-}}(S) \cup \sigma_{S B F_{+}^{-}}(T)$, but the two last inclusions are proper in general. After proving these results, we study the property $(g b)$ for direct sums. We give an example of operators $S \in L(X)$ and $T \in L(X)$ possessing $(g b)$ while their direct sum $S \oplus T$ does not possess property $(g b)$. Moreover, we explore certain sufficient conditions under which property $(g b)$ will be transferred from the direct summands to the direct sum. Thus in Theorem 2.6, we show that if $T$ and $S$ are Banach space operators possessing property $(g b)$, and if $\Pi(T) \subset$ $\sigma_{a}(S)$ and $\Pi(S) \subset \sigma_{a}(T)$, then $S \oplus T$ possesses property $(g b)$ if and only if $\sigma_{S B F_{+}^{-}}(S \oplus T)=\sigma_{S B F_{+}^{-}}(S) \cup \sigma_{S B F_{+}^{-}}(T)$. As a consequence, we show that if $T$ and $S$ are quasisimilar hyponormal operators possessing property $(g b)$, then $S \oplus T$ possesses property $(g b)$. Similarly, we show in Theorem 2.10 that if $T$ and $S$ both possesses property $(b)$, and if $\Pi^{0}(T) \subset \sigma_{a}(S)$ and $\Pi^{0}(S) \subset \sigma_{a}(T)$, then $S \oplus T$ possesses property $(b)$ if and only if $\sigma_{S F_{+}^{-}}(S \oplus T)=\sigma_{S F_{+}^{-}}(S) \cup \sigma_{S F_{+}^{-}}(T)$. In the third section, we prove in Theorem 3.1 that if $T \in L(X)$ and $S \in L(X)$ both possess property $(g w)$ and if $\sigma_{p}(S)=\sigma_{p}(T)$, then $S \oplus T$ possesses property $(g w)$ if and only if $\sigma_{S B F_{+}^{-}}(S \oplus T)=\sigma_{S B F_{+}^{-}}(S) \cup \sigma_{S B F_{+}^{-}}(T)$. We obtain a similar result in the case of property $(w)$.

\section{Preservation of properties $(b)$ and $(g b)$ under direct sums}

Let $T \in L(X)$ and $S \in L(X)$. In the first step we prove that the Drazin spectrum of a direct sum is the union of the Drazin spectra of its components. For this, we begin with the following auxiliary result that will be applied in the sequel.

Definition 2.1. Let $T \in L(X)$ and $S \in L(X)$. We will say that $T$ and $S$ are of stable sign index if for each $\lambda \notin \sigma_{S B F}(T)$ and $\mu \notin \sigma_{S B F}(S)$, ind $(T-$ 
$\lambda I)$ and $\operatorname{ind}(S-\mu I)$ have the same sign, where $\sigma_{S B F}(T)=\{\lambda \in \mathbb{C}: T-$ $\lambda I$ is not a semi-B-Fredholm operator $\}$.

Lemma 2.2. Let $T \in L(X)$ and $S \in L(X)$. Then

(i) $\sigma_{B F}(S \oplus T)=\sigma_{B F}(S) \cup \sigma_{B F}(T)$,

(ii) $\sigma_{B W}(S \oplus T) \subseteq \sigma_{B W}(S) \cup \sigma_{B W}(T)$,

(iii) $\sigma_{S B F_{+}^{-}}(S \oplus T) \subseteq \sigma_{S B F_{+}^{-}}(S) \cup \sigma_{S B F_{+}^{-}}(T)$.

Proof. (i) Let $\lambda \notin \sigma_{B F}(S) \cup \sigma_{B F}(T)$. Then $T-\lambda I$ and $S-\lambda I$ are both BFredholm operators. From [3, Theorem 2.7], each of $T-\lambda I$ and $S-\lambda I$ can be written as the direct sum of a Fredholm operator and a nilpotent operator, and the same is therefore true for the direct sum $S \oplus T-\lambda(I \oplus I)=(T-\lambda I) \oplus(S-\lambda I)$. Hence $S \oplus T-\lambda(I \oplus I)$ is a B-Fredholm operator and $\sigma_{B F}(S \oplus T) \subset \sigma_{B F}(S) \cup$ $\sigma_{B F}(T)$. Now if $\lambda \notin \sigma_{B F}(S \oplus T)$, we can assume without loss of generality that $\lambda=0$. Then $S \oplus T$ is a B-Fredholm operator. So there exists $n \in \mathbb{N}$ such that $R\left(S^{n}\right) \oplus R\left(T^{n}\right)$ is closed and $S_{[n]} \oplus T_{[n]}: R\left(S^{n}\right) \oplus R\left(T^{n}\right) \longrightarrow R\left(S^{n}\right) \oplus R\left(T^{n}\right)$ is a Fredholm operator. Consequently, $\alpha\left(T_{[n]}\right)<\infty, \beta\left(T_{[n]}\right)<\infty$ and $\alpha\left(S_{[n]}\right)<\infty$, $\beta\left(S_{[n]}\right)<\infty$. From [4, Theorem 3.1], $T$ and $S$ are B-Fredholm operators. Thus $0 \notin \sigma_{B F}(S) \cup \sigma_{B F}(T)$. Hence $\sigma_{B F}(S \oplus T)=\sigma_{B F}(S) \cup \sigma_{B F}(T)$.

(ii) If $\lambda \notin \sigma_{B W}(S) \cup \sigma_{B W}(T)$, without loss of generality we can assume that $\lambda=0$. Then $T$ and $S$ are both B-Weyl operators. Therefore there exists $n$ such that $R\left(T^{m}\right)$ and $R\left(S^{m}\right)$ are closed, $T_{[m]}$ and $S_{[m]}$ are Weyl operators for each $m \geq n$. Let $P: R\left(S^{m}\right) \oplus R\left(T^{m}\right) \longrightarrow R\left(S^{m}\right)$ be the linear projection along $R\left(T^{m}\right)$ onto $R\left(S^{m}\right)$. Then $P$ is continuous and this implies that $R\left(S^{m}\right) \oplus$ $R\left(T^{m}\right)=P^{-1}\left(R\left(S^{m}\right)\right)$ is closed. Since $\alpha\left(T_{[m]}\right)<\infty$ and $\alpha\left(S_{[m]}\right)<\infty$, we have $\alpha\left(S_{[m]} \oplus T_{[m]}\right)<\infty$. Since $R\left(T^{m+1}\right) \oplus R\left(S^{m+1}\right)$ is closed, $S_{[m]} \oplus T_{[m]}$ is an upper semi-Fredholm operator. It follows that $S \oplus T$ is an upper semi-B-Fredholm operator and $\operatorname{ind}(S \oplus T)=\operatorname{ind}(S)+\operatorname{ind}(T)=0$. So $S \oplus T$ is a B-Weyl operator and $0 \notin \sigma_{B W}(S \oplus T)$. Finally, we have $\sigma_{B W}(S \oplus T) \subseteq \sigma_{B W}(S) \cup \sigma_{B W}(T)$.

(iii) Follows in the same way as in (ii).

Generally, the inclusions (ii) and (iii) of Lemma 2.2 are proper (see example defined in Remark 2.7). Nonetheless, we give in the following proposition a sufficient condition under which the two inclusions become equalities.

Proposition 2.3. If $T \in L(X)$ and $S \in L(X)$ are of stable sign index, then

(i) $\sigma_{B W}(S \oplus T)=\sigma_{B W}(S) \cup \sigma_{B W}(T)$,

(ii) $\sigma_{S B F_{+}^{-}}(S \oplus T)=\sigma_{S B F_{+}^{-}}(S) \cup \sigma_{S B F_{+}^{-}}(T)$.

Proof. (i) Let $\alpha \notin \sigma_{B W}(S \oplus T)$ be arbitrary. Then $S \oplus T-\alpha(I \oplus I)$ is a B-Fredholm operator and $\operatorname{ind}(S \oplus T-\alpha(I \oplus I))=0$. From the assertion (i) of Lemma 2.2, $S-\alpha I$ and $T-\alpha I$ are B-Fredholm operators with ind $(S-\alpha I)+$ $\operatorname{ind}(T-\alpha I)=0$. As $T$ and $S$ are of stable sign index, then $\operatorname{ind}(S-\alpha I)=$ ind $(T-\alpha I)=0$ and so $\alpha \notin \sigma_{B W}(S) \cup \sigma_{B W}(T)$. Therefore $\sigma_{B W}(S) \cup \sigma_{B W}(T) \subset$ $\sigma_{B W}(S \oplus T)$ and since from Lemma $2.2 \sigma_{B W}(S) \cup \sigma_{B W}(T) \supset \sigma_{B W}(S \oplus T)$ is always true, it then follows that $\sigma_{B W}(S \oplus T)=\sigma_{B W}(S) \cup \sigma_{B W}(T)$. 
(ii) Let $\lambda \notin \sigma_{S B F_{+}^{-}}(S \oplus T)$ be arbitrary. We can assume without loss of generality that $\lambda=0$. Then $S \oplus T$ is an upper semi-B-Fredholm operator and $\operatorname{ind}(S \oplus T) \leq 0$. So there exists an integer $n$ for which $R\left(S^{n}\right) \oplus R\left(T^{n}\right)$ is closed and $S_{[n]} \oplus T_{[n]}: R\left(S^{n}\right) \oplus R\left(T^{n}\right) \longrightarrow R\left(S^{n}\right) \oplus R\left(T^{n}\right)$ is an upper semi-Fredholm operator. Hence $R\left(S^{n}\right)$ and $R\left(T^{n}\right)$ are closed and $S_{[n]}$ and $T_{[n]}$ are upper semi-Fredholm operators. Thus $T$ and $S$ are upper semi-BFredholm operators with $\operatorname{ind}(S \oplus T)=\operatorname{ind}(S)+\operatorname{ind}(T) \leq 0$. As $T$ and $S$ are of stable sign index, $\operatorname{ind}(S) \leq 0$ and $\operatorname{ind}(T) \leq 0$. This implies that $0 \notin$ $\sigma_{S B F_{+}^{-}}(S) \cup \sigma_{S B F_{+}^{-}}(T)$. Hence $\sigma_{S B F_{+}^{-}}(S) \cup \sigma_{S B F_{+}^{-}}(T) \subset \sigma_{S B F_{+}^{-}}(S \oplus T)$ and as it is always true that $\sigma_{S B F_{+}^{-}}(S) \cup \sigma_{S B F_{+}^{-}}(T) \supset \sigma_{S B F_{+}^{-}}(S \oplus T)$, we have $\sigma_{S B F_{+}^{-}}(S \oplus T)=\sigma_{S B F_{+}^{-}}(S) \cup \sigma_{S B F_{+}^{-}}(T)$.

Theorem 2.4. Let $T \in L(X)$ and $S \in L(X)$. Then $\sigma_{D}(T \oplus S)=\sigma_{D}(T) \cup$ $\sigma_{D}(S)$.

Proof. If $\lambda \notin \sigma_{D}(T) \cup \sigma_{D}(S)$, then $T-\lambda I$ and $S-\lambda I$ are Drazin invertible. Hence each of $T-\lambda I$ and $S-\lambda I$ can be written as the direct sum of an invertible operator and a nilpotent operator, and the same is therefore true for the direct sum $(T-\lambda I) \oplus(S-\lambda I)=T \oplus S-\lambda(I \oplus I)$. Thus $\lambda \notin \sigma_{D}(T \oplus S)$, and hence $\sigma_{D}(T \oplus S) \subset \sigma_{D}(T) \cup \sigma_{D}(S)$. Conversely, suppose that $\lambda \notin \sigma_{D}(T \oplus S)$. Then $T \oplus S-\lambda(I \oplus I)$ is a B-Fredholm operator of index 0 and $\lambda \in \operatorname{iso} \sigma(T \oplus S)$. From Lemma 2.2, $T-\lambda I$ and $S-\lambda I$ are both B-Fredholm operators. If $\lambda \notin \sigma(T)$, then $\lambda \notin \sigma_{D}(T)$. As $\lambda \in \operatorname{iso} \sigma(T \oplus S)$, then $\lambda \in$ iso $\sigma(S)$. By [4, Theorem 4.2], we deduce that $S-\lambda I$ is also Drazin invertible. Therefore $\lambda \notin \sigma_{D}(T) \cup \sigma_{D}(S)$. If $\lambda \in \sigma(T) \backslash \sigma(S)$, then $\lambda \in \operatorname{iso} \sigma(T)$ and again by [4, Theorem 4.2], $T-\lambda I$ is Drazin invertible. As $\lambda \notin \sigma(S)$, then $\lambda \notin \sigma_{D}(S)$. It then follows that $\lambda \notin \sigma_{D}(T) \cup \sigma_{D}(S)$. If $\lambda \in \sigma(T) \cap \sigma(S)$, then $\lambda \in$ iso $\sigma(T) \cap$ iso $\sigma(S)$. Hence $\lambda \notin \sigma_{D}(T) \cup \sigma_{D}(S)$. In the two cases we have $\sigma_{D}(T) \cup \sigma_{D}(S) \subset \sigma_{D}(T \oplus S)$. Hence $\sigma_{D}(T \oplus S)=\sigma_{D}(T) \cup \sigma_{D}(S)$.

Generally, if $T \in L(X)$ and $S \in L(X)$ both possess property $(g b)$, then it is not guaranteed that their (orthogonal) direct sum $S \oplus T \in L(X \oplus X)$ possesses property $(g b)$, as shown by the following example.

Example 2.5. Let $S=0$ and $R$ be the unilateral right shift operator defined on the Hilbert space $\ell^{2}(\mathbb{N})$. Then $\sigma(S)=\sigma_{a}(S)=\{0\}, \sigma_{S B F_{+}}(S)=\emptyset, \Pi(S)=$ $\{0\}$. Thus $\sigma_{a}(S) \backslash \sigma_{S B F_{+}^{-}}(S)=\Pi(S)$ and $S$ possesses property $(g b)$. Moreover, $\sigma(R)=D(0,1)$ is the closed unit disc in $\mathbb{C}, \sigma_{a}(R)=C(0,1)$ is the unit circle of $\mathbb{C}, \sigma_{S B F_{+}^{-}}(R)=C(0,1)$, and $\Pi(R)=\emptyset$. Therefore $\sigma_{a}(R) \backslash \sigma_{S B F_{+}^{-}}(R)=\Pi(R)$ and $R$ possesses property $(g b)$. But the operator $T$ defined on the Banach space $\ell^{2}(\mathbb{N}) \oplus \ell^{2}(\mathbb{N})$ by $T=S \oplus R$ does not possess property $(g b)$, because $\sigma_{a}(T)=C(0,1) \cup\{0\}, \sigma_{S B F_{+}^{-}}(T)=C(0,1)$, and $\Pi(T)=\emptyset$.

However, we provide, in the following result, certain conditions on $T$ and $S$ to ensure that their orthogonal direct sum $S \oplus T$ possesses property $(g b)$. 
Theorem 2.6. Suppose that $T \in L(X)$ and $S \in L(X)$ be such that $\Pi(T) \subset$ $\sigma_{a}(S)$ and $\Pi(S) \subset \sigma_{a}(T)$. If $T$ and $S$ both possess property $(g b)$, then the following statements are equivalent.

(i) $S \oplus T$ possesses property $(g b)$,

(ii) $\sigma_{S B F_{+}^{-}}(S \oplus T)=\sigma_{S B F_{+}^{-}}(S) \cup \sigma_{S B F_{+}^{-}}(T)$.

Proof. (ii) $\Rightarrow$ (i) Assume that $\sigma_{S B F_{+}^{-}}(S \oplus T)=\sigma_{S B F_{+}^{-}}(S) \cup \sigma_{S B F_{+}^{-}}(T)$. We know that $\sigma(S \oplus T)=\sigma(S) \cup \sigma(T)$ for every pair of operators, and from Theorem 2.4, we have $\sigma_{D}(S \oplus T)=\sigma_{D}(S) \cup \sigma_{D}(T)$. Hence

$$
\begin{aligned}
\Pi(S \oplus T) & =\sigma(S \oplus T) \backslash \sigma_{D}(S \oplus T) \\
& =[\sigma(S) \cup \sigma(T)] \backslash\left[\sigma_{D}(S) \cup \sigma_{D}(T)\right] \\
& =[\Pi(S) \cap \rho(T)] \cup[\Pi(T) \cap \rho(S)] \cup[\Pi(S) \cap \Pi(T)] .
\end{aligned}
$$

By hypothesis $\Pi(T) \subset \sigma_{a}(S)$ and $\Pi(S) \subset \sigma_{a}(T), \Pi(T) \cap \rho(S)=\emptyset$ and $\Pi(S) \cap$ $\rho(T)=\emptyset$. Hence $\Pi(S \oplus T)=\Pi(S) \cap \Pi(T)$. On the other hand, since $T$ and $S$ both possess property $(g b)$, then

$$
\begin{aligned}
& {\left[\sigma_{a}(S) \cup \sigma_{a}(T)\right] \backslash\left[\sigma_{S B F_{+}^{-}}(S) \cup \sigma_{S B F_{+}^{-}}(T)\right] } \\
= & {\left[\Pi(S) \cap \rho_{a}(T)\right] \cup\left[\Pi(T) \cap \rho_{a}(S)\right] \cup[\Pi(S) \cap \Pi(T)] . }
\end{aligned}
$$

Again by hypothesis we have $\Pi(S) \cap \rho_{a}(T)=\emptyset$ and $\Pi(T) \cap \rho_{a}(S)=\emptyset$. Thus,

$$
\left[\sigma_{a}(S) \cup \sigma_{a}(T)\right] \backslash\left[\sigma_{S B F_{+}^{-}}(S) \cup \sigma_{S B F_{+}^{-}}(T)\right]=\Pi(S \oplus T) .
$$

We know that $\sigma_{a}(S \oplus T)=\sigma_{a}(S) \cup \sigma_{a}(T)$ for any pair of operators and by assumption, $\sigma_{S B F_{+}^{-}}(S \oplus T)=\sigma_{S B F_{+}^{-}}(S) \cup \sigma_{S B F_{+}^{-}}(T)$. Hence $\sigma_{a}(S \oplus T) \backslash$

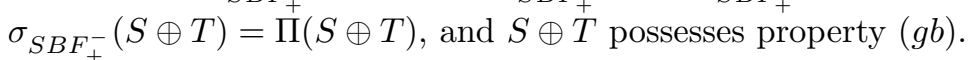

(i) $\Rightarrow$ (ii) If $S \oplus T$ possesses property $(g b)$, then $\sigma_{S B F_{+}^{-}}(S \oplus T)=\sigma_{a}(S \oplus$ $T) \backslash \Pi(S \oplus T)$. From the equality (2.2) we have $\sigma_{S B F_{+}^{-}}(S) \cup \sigma_{S B F_{+}^{-}}(T)=$ $\sigma_{a}(S \oplus T) \backslash \Pi(S \oplus T)$. So $\sigma_{S B F_{+}^{-}}(S \oplus T)=\sigma_{S B F_{+}^{-}}(S) \cup \sigma_{S B F_{+}^{-}}(T)$.

Remark 2.7. The identity $\sigma_{S B F_{+}^{-}}(S \oplus T)=\sigma_{S B F_{+}^{-}}(S) \cup \sigma_{S B F_{+}^{-}}(T)$ assumed in Theorem 2.6 plays a central role in establishing conditions for the direct sum to possess property $(g b)$. For this, define on the Banach space $\ell^{2}(\mathbb{N}) \oplus$ $\ell^{2}(\mathbb{N})$ the operator $R \oplus L$, where $R$ and $L$ denote the right shift operator and the left shift operator, respectively, on the Hilbert space $\ell^{2}(\mathbb{N})$. Then $R$ possesses property $(g b)$. On the other hand, $L$ possesses property $(g b)$, since $\sigma_{a}(L)=D(0,1), \sigma_{S B F_{+}^{-}}(L)=D(0,1)$, and $\Pi(L)=\emptyset$. Moreover, $\Pi(R) \subset \sigma_{a}(L)$ and $\Pi(L) \subset \sigma_{a}(R)$. But $R \oplus L$ does not possess property ( $\left.g b\right)$. Indeed, as $\alpha(R \oplus L)=\beta(R \oplus L)=1,0 \notin \sigma_{S B F_{+}^{-}}(R \oplus L)$, and since $a(R \oplus L)=\infty$, it then follows from [5, Theorem 2.5] that $R \oplus L$ does not have the SVEP at 0 . Consequently, $R \oplus L$ does not satisfy generalized a-Browder's theorem. From [8, Corollary 2.8], $R \oplus L$ does not possess property $(g b)$. Observe that 
$\sigma_{S B F_{+}^{-}}(R \oplus L) \neq \sigma_{S B F_{+}^{-}}(R) \cup \sigma_{S B F_{+}^{-}}(L)$, because $\sigma_{S B F_{+}^{-}}(R) \cup \sigma_{S B F_{+}^{-}}(L)=$ $D(0,1)$ and $0 \notin \sigma_{S B F_{+}^{-}}(R \oplus L)$.

The (bounded linear) operator $A: X \longrightarrow Y$ is said to be quasi-invertible if it is injective and has dense range. Two bounded linear operators $T \in L(X)$ and $S \in L(Y)$ on complex Banach spaces $X$ and $Y$ are quasisimilar provided there exist quasi-invertible operators $A: X \longrightarrow Y$ and $B: Y \longrightarrow X$ such that $A T=S A$ and $B S=T B$. In this case the operators $\tilde{T}_{[n]}: \overline{R\left(T^{n}\right)} \longrightarrow \overline{R\left(T^{n}\right)}$ and $\tilde{S}_{[n]}: \overline{R\left(S^{n}\right)} \longrightarrow \overline{R\left(S^{n}\right)}$ are also quasisimilar. Indeed, if we consider $P=A / \overline{R\left(T^{n}\right)}: \overline{R\left(T^{n}\right)} \longrightarrow \overline{R\left(S^{n}\right)}$ and $Q=B / \overline{R\left(S^{n}\right)}: \overline{R\left(S^{n}\right)} \longrightarrow \overline{R\left(T^{n}\right)}$, then it is easily seen that $\overline{P\left(\overline{R\left(T^{n}\right)}\right)}=\overline{R\left(S^{n}\right)}, \overline{Q\left(\overline{R\left(S^{n}\right)}\right)}=\overline{R\left(T^{n}\right)}, P$ and $Q$ are both injective, $P \tilde{T}_{[n]}=\tilde{S}_{[n]} P$, and $Q \tilde{S}_{[n]}=\tilde{T}_{[n]} Q$.

A bounded linear operator $T \in L(H)$ on a Hilbert space $H$ is said to be hyponormal if $T^{*} T-T T^{*} \geq 0$ (or equivalently $\left\|T^{*} x\right\| \leq\|T x\|$ for all $x \in H$ ).

Lemma 2.8. If $T \in L(H)$ and $S \in L(H)$ are quasisimilar hyponormal operators, then $\Pi(T)=\Pi(S)$.

Proof. Let $\alpha \in \Pi(T)$ be arbitrary. Then $T-\alpha I$ is Drazin invertible and $a(T-\alpha I)=\delta(T-\alpha I)=n<\infty$. Without loss of generality we may assume that $\alpha=0$. Therefore $c_{n}(T)=c_{n}^{\prime}(T)=0$ and $R\left(T^{n}\right)$ is closed. As $c_{n}(T)=$ $c_{0}\left(T_{[n]}\right)$ and $c_{n}^{\prime}(T)=c_{0}^{\prime}\left(T_{[n]}\right)$, then $T_{[n]}: R\left(T^{n}\right) \longrightarrow R\left(T^{n}\right)$ is invertible. On the other hand, $T_{[n]}: R\left(T^{n}\right) \longrightarrow R\left(T^{n}\right)$ and $\tilde{S}_{[n]}: \overline{R\left(S^{n}\right)} \longrightarrow \overline{R\left(S^{n}\right)}$ are hyponormal quasisimilar operators. Since $T_{[n]}$ is invertible, from [9, Theorem 1] $\tilde{S}_{[n]}$ is invertible. So $\left(\tilde{S}_{[n]}\right)^{n}$ is invertible and $R\left(\left(\tilde{S}_{[n]}\right)^{n}\right)=\overline{R\left(S^{n}\right)}$. As $R\left(\left(\tilde{S}_{[n]}\right)^{n}\right) \subseteq R\left(S^{n}\right)$, then $\overline{R\left(S^{n}\right)}=R\left(S^{n}\right)$, i.e., $R\left(S^{n}\right)$ is closed. Therefore $0 \notin \sigma_{D}(S)$. As we know from [9, Theorem 1] that $\sigma(T)=\sigma(S)$, then $0 \in \Pi(S)$. Similarly, we have $\Pi(S) \subset \Pi(T)$. Hence $\Pi(T)=\Pi(S)$.

Corollary 2.9. Let $T \in L(H)$ and $S \in L(H)$ are quasisimilar hyponormal operators. If $T$ and $S$ both possesses property $(g b)$, then $S \oplus T$ possesses property $(g b)$.

Proof. As it is well known that every hyponormal operator has the SVEP, then from [5, Theorem 2.5], we conclude that ind $(T-\lambda I) \leq 0$ and ind $(S-\mu I) \leq 0$ for each $\lambda \in \rho_{S B F}(T)$ and $\mu \in \rho_{S B F}(S)$. From Proposition 2.3, it follows that $\sigma_{S B F_{+}^{-}}(S \oplus T)=\sigma_{S B F_{+}^{-}}(S) \cup \sigma_{S B F_{+}^{-}}(T)$. As $\Pi(T)=\Pi(S)$ (see Lemma 2.8), then $\Pi(T) \subset \sigma_{a}(S)$ and $\Pi(S) \subset \sigma_{a}(T)$ and since $T$ and $S$ both possess property $(g b)$, then by Theorem 2.6, $S \oplus T$ possesses property $(g b)$.

Similarly to Theorem 2.6, we give the following characterization in the case of property $(b)$.

Theorem 2.10. Suppose that property (b) holds for $T \in L(X)$ and $S \in L(X)$. If $\Pi^{0}(T) \subset \sigma_{a}(S)$ and $\Pi^{0}(S) \subset \sigma_{a}(T)$, then the following assertions are equivalent. 
(i) $S \oplus T$ possesses property $(b)$,

(ii) $\sigma_{S F_{+}^{-}}(S \oplus T)=\sigma_{S F_{+}^{-}}(S) \cup \sigma_{S F_{+}^{-}}(T)$.

Proof. (ii) $\Rightarrow$ (i) Suppose that $\sigma_{S F_{+}^{-}}(S \oplus T)=\sigma_{S F_{+}^{-}}(S) \cup \sigma_{S F_{+}^{-}}(T)$. Since $T$ and $S$ both possess property $(b)$, then

$$
\begin{aligned}
& {\left[\sigma_{a}(S) \cup \sigma_{a}(T)\right] \backslash\left[\sigma_{S F_{+}^{-}}(S) \cup \sigma_{S F_{+}^{-}}(T)\right] } \\
= & {\left[\Pi^{0}(S) \cap \rho_{a}(T)\right] \cup\left[\Pi^{0}(T) \cap \rho_{a}(S)\right] \cup\left[\Pi^{0}(S) \cap \Pi^{0}(T)\right] . }
\end{aligned}
$$

The assumption $\Pi^{0}(T) \subset \sigma_{a}(S)$ and $\Pi^{0}(S) \subset \sigma_{a}(T)$ entails that $\Pi^{0}(T) \cap$ $\rho_{a}(S)=\emptyset$ and $\Pi^{0}(S) \cap \rho_{a}(T)=\emptyset$. Therefore $\left[\sigma_{a}(S) \cup \sigma_{a}(T)\right] \backslash\left[\sigma_{S F_{+}^{-}}(S) \cup\right.$ $\left.\sigma_{S F_{+}^{-}}(T)\right]=\Pi^{0}(S) \cap \Pi^{0}(T)$. On the other hand, as we know that $\sigma_{b}(S \oplus T)=$ $\sigma_{b}(S) \cup \sigma_{b}(T)$ for any pair of operators, then

$$
\begin{aligned}
\Pi^{0}(S \oplus T) & =\sigma(S \oplus T) \backslash \sigma_{b}(S \oplus T) \\
& =[\sigma(S) \cup \sigma(T)] \backslash\left[\sigma_{b}(S) \cup \sigma_{b}(T)\right] \\
& =\left[\Pi^{0}(S) \cap \rho(T)\right] \cup\left[\Pi^{0}(T) \cap \rho(S)\right] \cup\left[\Pi^{0}(S) \cap \Pi^{0}(T)\right] \\
& =\Pi^{0}(S) \cap \Pi^{0}(T), \text { since } \Pi^{0}(S) \cap \rho(T)=\Pi^{0}(T) \cap \rho(S)=\emptyset .
\end{aligned}
$$

Thus

$$
\left.\Pi^{0}(S \oplus T)=\sigma_{a}(S) \cup \sigma_{a}(T)\right] \backslash\left[\sigma_{S F_{+}^{-}}(S) \cup \sigma_{S F_{+}^{-}}(T)\right] .
$$

By hypothesis $\sigma_{S F_{+}^{-}}(S \oplus T)=\sigma_{S F_{+}^{-}}(S) \cup \sigma_{S F_{+}^{-}}(T)$, it then follows that $\Pi^{0}(S \oplus$ $T)=\sigma_{a}(S \oplus T) \backslash \sigma_{S F_{+}^{-}}(S \oplus T)$ and $S \oplus T$ possesses property $(b)$.

(i) $\Rightarrow$ (ii) If $S \oplus T$ possesses property $(b)$, then $\sigma_{S F_{+}^{-}}(S \oplus T)=\sigma_{a}(S \oplus T) \backslash$ $\Pi^{0}(S \oplus T)$. From the equality (2.3), it then follows that $\sigma_{S F_{+}^{-}}(S) \cup \sigma_{S F_{+}^{-}}(T)=$ $\sigma_{a}(S \oplus T) \backslash \Pi^{0}(S \oplus T)$. Hence $\sigma_{S F_{+}^{-}}(S \oplus T)=\sigma_{S F_{+}^{-}}(S) \cup \sigma_{S F_{+}^{-}}(T)$.

The following examples show that if $S$ and $T$ are Banach space operators possessing property $(b)$, then it does not necessarily imply that the direct sum $S \oplus T$ possesses property $(b)$. Moreover, the first example (part (1)) shows that $\sigma_{S F_{+}^{-}}(S \oplus T)=\sigma_{S F_{+}^{-}}(S) \cup \sigma_{S F_{+}^{-}}(T)$ is not a sufficient condition on the direct sum to possess property $(b)$, but the second example (part (2)) shows that the symmetric assumption $\Pi^{0}(S) \subset \sigma_{a}(T), \Pi^{0}(T) \subset \sigma_{a}(S)$ on the direct summands is not a sufficient condition to ensure that the direct sum possesses property $(b)$.

Example 2.11. (1) Let us define the operator $S$ on the Hilbert space $\ell^{2}(\mathbb{N})$ by $S\left(x_{1}, x_{2}, x_{3}, \ldots\right)=\left(0, x_{2}, x_{3}, x_{4}, \ldots\right)$. Then $\sigma(S)=\sigma_{a}(S)=\{0,1\}, \sigma_{S F_{+}^{-}}(S)=$ $\{1\}$, and $\Pi^{0}(S)=\{0\}$. Thus $\sigma_{a}(S) \backslash \sigma_{S F_{+}^{-}}(S)=\Pi^{0}(S)$ and $S$ possesses property (b). Now, we consider the unilateral right shift operator $R$ defined on $\ell^{2}(\mathbb{N})$, then $R$ possesses property $(b)$, since $\sigma_{a}(R)=C(0,1), \sigma_{S F_{+}^{-}}(R)=C(0,1)$, and $\Pi^{0}(R)=\emptyset$. Define $T$ on the Banach space $X=\ell^{2}(\mathbb{N}) \oplus \ell^{2}(\mathbb{N})$ by $T=R \oplus S$. 
Then $\sigma_{a}(T)=C(0,1) \cup\{0\}, \sigma_{S F_{+}^{-}}(T)=C(0,1)$, and $\Pi^{0}(T)=\emptyset$. Hence $\sigma_{a}(T) \backslash \sigma_{S F_{+}^{-}}(T) \neq \Pi^{0}(T)$, and $T$ does not possess property $(b)$. Note that $\Pi^{0}(R) \subset \sigma_{a}(S), \Pi^{0}(S) \not \subset \sigma_{a}(R)$, and $\sigma_{S F_{+}^{-}}(R \oplus S)=\sigma_{S F_{+}^{-}}(R) \cup \sigma_{S F_{+}^{-}}(S)$.

(2) In Theorem 2.10, the condition $\sigma_{S F_{+}^{-}}(S \oplus T)=\sigma_{S F_{+}^{-}}(S) \cup \sigma_{S F_{+}^{-}}(T)$ is crucial. Indeed, if we consider the operator $T=R \oplus L$ defined as in Remark 2.7, then $R$ possesses property $(b)$ because $\sigma_{a}(R)=C(0,1), \sigma_{S F_{+}^{-}}(R)=C(0,1)$, and $\Pi^{0}(R)=\emptyset$. Its adjoint $L$ possesses property $(b)$ because $\sigma_{a}(L)=D(0,1)$, $\sigma_{S F_{+}^{-}}(L)=D(0,1)$, and $\Pi^{0}(L)=\emptyset$. Moreover, $\Pi^{0}(R) \subset \sigma_{a}(L)$ and $\Pi^{0}(L) \subset$ $\sigma_{a}(R)$. But, since $0 \notin \sigma_{S F_{+}^{-}}(R \oplus L)$ and $R \oplus L$ does not have the SVEP at 0 , it then follows that $R \oplus L$ does not satisfy a-Browder's theorem. By [8, Theorem 2.5], $R \oplus L$ does not possess property $(b)$. Note that the inclusion $\sigma_{S F_{+}^{-}}(R \oplus L) \subset \sigma_{S F_{+}^{-}}(R) \cup \sigma_{S F_{+}^{-}}(L)$ is proper, since $\sigma_{S F_{+}^{-}}(R) \cup \sigma_{S F_{+}^{-}}(L)=$ $D(0,1)$ and $0 \notin \sigma_{S F_{+}^{-}}(R \oplus L)$.

As for every quasisimilar hyponormal operators $T \in L(H)$ and $S \in L(H)$, we have $\alpha(T-\lambda I)=\alpha(S-\lambda I)$ for all $\lambda \in \mathbb{C}$, then $\Pi^{0}(S)=\Pi^{0}(T)$. From Theorem 2.10, we obtain immediately the following corollary.

Corollary 2.12. Let $T \in L(H)$ and $S \in L(H)$ be quasisimilar hyponormal operators. If $T$ and $S$ both possess property (b), then $S \oplus T$ possesses property (b).

The following example shows that there exist $T \in L(X)$ and $S \in L(X)$ such that their (orthogonal) direct sum $S \oplus T$ does not satisfy generalized Browder's theorem, although $T$ and $S$ both satisfy generalized Browder's theorem. We consider the operator $T=R \oplus L$ as defined in Remark 2.7, then $\sigma(R)=$ $D(0,1), \sigma_{B W}(R)=D(0,1)$, and $\Pi(R)=\emptyset$. So $R$ satisfies generalized Browder's theorem. On the other hand, as $\sigma(L)=D(0,1), \sigma_{B W}(R)=D(0,1)$, and $\Pi(L)=\emptyset$ then $L$ satisfies generalized Browder's theorem. But $T=R \oplus L$ does not satisfy generalized Browder's theorem. Indeed, as $\alpha(R \oplus L)=\beta(R \oplus L)=$ 1 then $0 \notin \sigma_{B W}(R \oplus L)$ and, since $R \oplus L$ does not have the SVEP at 0 , it then follows that $R \oplus L$ does not satisfy generalized Browder's theorem. Note that the inclusion $\sigma_{B W}(R \oplus L) \subset \sigma_{B W}(R) \cup \sigma_{B W}(L)$ is proper, because $\sigma_{B W}(R) \cup \sigma_{B W}(L)=D(0,1)$ and $0 \notin \sigma_{B W}(R \oplus L)$.

In [13, Theorem 3.6], it is established that if $S$ and $T$ are polaroid operators acting on Hilbert spaces, satisfying generalized Browder's theorem and if $\sigma_{B W}(S) \cup \sigma_{B W}(T)=\sigma_{B W}(S \oplus T)$, then $S \oplus T$ satisfies generalized Browder's theorem. Using Theorem 2.4, we can extend this result as follows.

Theorem 2.13. Let $T \in L(X)$ and $S \in L(X)$. If generalized Browder's theorem holds for $T$ and $S$, then the following statements are equivalent.

(i) $S \oplus T$ satisfies generalized Browder's theorem,

(ii) $\sigma_{B W}(S) \cup \sigma_{B W}(T)=\sigma_{B W}(S \oplus T)$. 
Proof. (i) $\Rightarrow$ (ii) If generalized Browder's theorem holds for $S \oplus T$, then $\sigma_{B W}(S \oplus$ $T)=\sigma_{D}(S \oplus T)$. As $\sigma_{D}(S \oplus T)=\sigma_{D}(S) \cup \sigma_{D}(T)$ (see Theorem 2.4), then $\sigma_{B W}(S \oplus T)=\sigma_{D}(S) \cup \sigma_{D}(T)$. Since we have always $\sigma_{B W}(S) \cup \sigma_{B W}(T) \subset$ $\sigma_{D}(S) \cup \sigma_{D}(T)$, it then follows that $\sigma_{B W}(S) \cup \sigma_{B W}(T) \subset \sigma_{B W}(S \oplus T)$. By virtue of Lemma 2.2 we conclude that $\sigma_{B W}(S) \cup \sigma_{B W}(T)=\sigma_{B W}(S \oplus T)$.

(ii) $\Rightarrow$ (i) Assume that $\sigma_{B W}(S) \cup \sigma_{B W}(T)=\sigma_{B W}(S \oplus T)$. Since $T$ and $S$ both satisfy generalized Browder's theorem then $\sigma_{B W}(T)=\sigma_{D}(T)$ and $\sigma_{B W}(S)=$ $\sigma_{D}(S)$. Therefore $\sigma_{D}(S \oplus T)=\sigma_{D}(S) \cup \sigma_{D}(T)=\sigma_{B W}(S) \cup \sigma_{B W}(T)$. By hypothesis $\sigma_{B W}(S) \cup \sigma_{B W}(T)=\sigma_{B W}(S \oplus T), \sigma_{D}(S \oplus T)=\sigma_{B W}(S \oplus T)$ and $S \oplus T$ satisfies generalized Browder's theorem.

\section{Preservation of properties $(w)$ and $(g w)$ under direct sums}

Theorem 3.1. Suppose that $T \in L(X)$ and $S \in L(X)$ be such that $\sigma_{p}(T)=$ $\sigma_{p}(S)$. If $T$ and $S$ both possess property $(g w)$, then the following assertions are equivalent.

(i) $S \oplus T$ possesses property $(g w)$,

(ii) $\sigma_{S B F_{+}^{-}}(S \oplus T)=\sigma_{S B F_{+}^{-}}(S) \cup \sigma_{S B F_{+}^{-}}(T)$.

Proof. (ii) $\Rightarrow$ (i) Suppose that $\sigma_{S B F_{+}^{-}}(S \oplus T)=\sigma_{S B F_{+}^{-}}(S) \cup \sigma_{S B F_{+}^{-}}(T)$. Since $T$ and $S$ both possess property $(g w)$, we have

$$
\begin{aligned}
& \sigma_{a}(S \oplus T) \backslash \sigma_{S B F_{+}^{-}}(S \oplus T) \\
= & {\left[\sigma_{a}(S) \cup \sigma_{a}(T)\right] \backslash\left[\sigma_{S B F_{+}^{-}}(S) \cup \sigma_{S B F_{+}^{-}}(T)\right] } \\
= & {\left[E(T) \cap \rho_{a}(S)\right] \cup\left[E(S) \cap \rho_{a}(T)\right] \cup[E(S) \cap E(T)] . }
\end{aligned}
$$

As $\sigma_{p}(S)=\sigma_{p}(T)$, then $E(T) \cap \rho_{a}(S)=\emptyset$ and $E(S) \cap \rho_{a}(T)=\emptyset$. Thus $\sigma_{a}(S \oplus T) \backslash \sigma_{S B F_{+}^{-}}(S \oplus T)=E(S) \cap E(T)$. On the other hand, we have

$$
E(S \oplus T)
$$$$
=\operatorname{iso} \sigma(S \oplus T) \cap \sigma_{p}(S \oplus T)
$$

$(3.2)=\operatorname{iso}[\sigma(S) \cup \sigma(T)] \cap\left[\sigma_{p}(S) \cup \sigma_{p}(T)\right]$

$=[E(S) \cap \rho(T)] \cup[E(T) \cap \rho(S)] \cup[E(S) \cap$ iso $\sigma(T)] \cup[E(T) \cap$ iso $\sigma(S)]$

$=E(S) \cap E(T)$, since $E(S) \cap \rho(T)=\emptyset$ and $E(T) \cap \rho(S)=\emptyset$.

Hence $E(S \oplus T)=\sigma_{a}(S \oplus T) \backslash \sigma_{S B F_{+}^{-}}(S \oplus T)$ and $S \oplus T$ possesses property $(g w)$.

(i) $\Rightarrow$ (ii) If $S \oplus T$ possesses property $(g w)$, then $\sigma_{a}(S \oplus T) \backslash \sigma_{S B F_{+}^{-}}(S \oplus T)=$ $E(S \oplus T)$. Since $T$ and $S$ both possess property $(g w)$ and $\sigma_{p}(T) \stackrel{+}{=} \sigma_{p}(S)$, it then follows from the equality (3.1) that $\sigma_{a}(S \oplus T) \backslash\left[\sigma_{S B F_{+}^{-}}(S) \cup \sigma_{S B F_{+}^{-}}(T)\right]=$ $E(S) \cap E(T)$ and from the equality (3.2) that $E(S \oplus T)=E(S) \cap E(T)$. Hence $\sigma_{S B F_{+}^{-}}(S \oplus T)=\sigma_{S B F_{+}^{-}}(S) \cup \sigma_{S B F_{+}^{-}}(T)$. 
Remark 3.2. If $T$ and $S$ are Banach space operators possessing property ( $g w)$, then it does not necessarily imply that the direct sum $S \oplus T$ possesses property $(g w)$. For example, on the Hilbert space $\ell^{2}(\mathbb{N})$ we consider the operators $S=$ 0 and $U$ defined by $U\left(x_{1}, x_{2}, x_{3}, \ldots\right)=\left(0, x_{1} / 2, x_{2} / 3, \ldots\right)$. Then $\sigma_{a}(S)=$ $\{0\}, \sigma_{S B F_{+}^{-}}(S)=\emptyset$, and $E(S)=\{0\}$. Thus $\sigma_{a}(S) \backslash \sigma_{S B F_{+}^{-}}(S)=E(S)$, and so $S$ possesses property $(g w)$. On the other hand, we have $\sigma_{a}(U)=\{0\}$, $\sigma_{S B F_{+}^{-}}(U)=\{0\}$ and $E(U)=\emptyset$. Therefore $\sigma_{a}(U) \backslash \sigma_{S B F_{+}^{-}}(U)=E(U)$ and so $U$ possesses property $(g w)$. We define $T$ on the Banach space $\ell^{2}(\mathbb{N}) \oplus \ell^{2}(\mathbb{N})$ by $T=S \oplus U$. Then $\sigma_{a}(T)=\{0\}, \sigma_{S B F_{+}^{-}}(T)=\{0\}$, and $E(T)=\{0\}$. Therefore $\sigma_{a}(T) \backslash \sigma_{S B F_{+}^{-}}(T) \neq E(T)$ and so $T$ does not possess property $(g w)$. Note that $\sigma_{p}(S)=\{0\}, \sigma_{p}(U)=\emptyset$, and $\sigma_{S B F_{+}^{-}}(S \oplus U)=\sigma_{S B F_{+}^{-}}(S) \cup \sigma_{S B F_{+}^{-}}(U)$. $(w)$.

Similarly in Theorem 3.1, we have the following result in the case of property

Theorem 3.3. Suppose that $T \in L(X)$ and $S \in L(X)$ be such that $\sigma_{p}^{0}(T)=$ $\sigma_{p}^{0}(S)$. If $T$ and $S$ both possess property $(w)$, then the following statements are equivalent.

(i) $S \oplus T$ possesses property $(w)$,

(ii) $\sigma_{S F_{+}^{-}}(S \oplus T)=\sigma_{S F_{+}^{-}}(S) \cup \sigma_{S F_{+}^{-}}(T)$.

Proof. (ii) $\Rightarrow$ (i) Suppose that $\sigma_{S F_{+}^{-}}(S \oplus T)=\sigma_{S F_{+}^{-}}(S) \cup \sigma_{S F_{+}^{-}}(T)$. Since $T$ and $S$ both possess property $(w)$, we have

$$
\begin{aligned}
& \sigma_{a}(S \oplus T) \backslash \sigma_{S F_{+}^{-}}(S \oplus T) \\
= & {\left[\sigma_{a}(S) \cup \sigma_{a}(T)\right] \backslash\left[\sigma_{S F_{+}^{-}}(S) \cup \sigma_{S F_{+}^{-}}(T)\right] } \\
= & {\left[E^{0}(T) \cap \rho_{a}(S)\right] \cup\left[E^{0}(S) \cap \rho_{a}(T)\right] \cup\left[E^{0}(S) \cap E^{0}(T)\right] . }
\end{aligned}
$$

As $\sigma_{p}^{0}(S)=\sigma_{p}^{0}(T)$, then $E^{0}(T) \cap \rho_{a}(S)=\emptyset$ and $E^{0}(S) \cap \rho_{a}(T)=\emptyset$. Thus $\sigma_{a}(S \oplus T) \backslash \sigma_{S F_{+}^{-}}(S \oplus T)=E^{0}(S) \cap E^{0}(T)$. On the other hand, since $\sigma_{p}^{0}(S \oplus T)=$ $\left\{\lambda \in \sigma_{p}^{0}(S) \cup \sigma_{p}^{0}(T): \operatorname{dim} N(S-\lambda I)+\operatorname{dim} N(T-\lambda I)<\infty\right\}$, we have

$$
E^{0}(S \oplus T)
$$$$
=\operatorname{iso} \sigma(S \oplus T) \cap \sigma_{p}^{0}(S \oplus T)
$$$$
=\operatorname{iso}[\sigma(S) \cup \sigma(T)] \cap\left[\sigma_{p}^{0}(S) \cup \sigma_{p}^{0}(T)\right]
$$$$
=\operatorname{iso}[\sigma(S) \cup \sigma(T)] \cap \sigma_{p}^{0}(S)
$$$$
=\left[E^{0}(S) \cap \rho(T)\right] \cup\left[E^{0}(T) \cap \rho(S)\right] \cup\left[E^{0}(S) \cap E^{0}(T)\right]
$$$$
=E^{0}(S) \cap E^{0}(T) \text {, since } E^{0}(S) \cap \rho(T)=\emptyset \text { and } E^{0}(T) \cap \rho(S)=\emptyset \text {. }
$$

Hence $\sigma_{a}(S \oplus T) \backslash \sigma_{S F_{+}^{-}}(S \oplus T)=E^{0}(S \oplus T)$ and $S \oplus T$ possess property $(w)$. (i) $\Rightarrow$ (ii) Assume that $S \oplus T$ possesses property $(w)$, then $\sigma_{a}(S \oplus T) \backslash \sigma_{S F_{+}^{-}}(S \oplus$ $T)=E^{0}(S \oplus T)$. As $T$ and $S$ both possess property $(w)$ and have the same 
eigenvalues of finite multiplicity, it then follows from the equality (3.3) that $\sigma_{a}(S \oplus T) \backslash\left[\sigma_{S F_{+}^{-}}(S) \cup \sigma_{S F_{+}^{-}}(T)\right]=E^{0}(S) \cap E^{0}(T)$ and by the equality (3.4) that $E^{0}(S) \cap E^{0}(T)=E^{0}(S \oplus T)$. Hence $\sigma_{S F_{+}^{-}}(S \oplus T)=\sigma_{S F_{+}^{-}}(S) \cup \sigma_{S F_{+}^{-}}(T)$.

Remark 3.4. There are Banach space operators $T$ and $S$ both of which possess property $(w)$, but their direct sum $S \oplus T$ does not possess property $(w)$. Indeed, if we consider the operator $T=R \oplus S$ defined as in Example 2.11, then $\sigma_{a}(R)=$ $\sigma_{S F_{+}^{-}}(R)=C(0,1)$ and $E^{0}(R)=\emptyset$. So $R$ possesses property $(w)$. The operator $S$ also possesses property $(w)$, since $\sigma_{a}(S)=\{0,1\}, \sigma_{S F_{+}^{-}}(S)=\{1\}$, and $E^{0}(S)=\{0\}$. But the direct sum $T=R \oplus S$ does not possess property $(w)$, because $\sigma_{a}(T)=C(0,1) \cup\{0\}, \sigma_{S F_{+}^{-}}(T)=C(0,1)$, and $E^{0}(T)=\emptyset$. Note that $\sigma_{p}^{0}(S)=\{0\}, \sigma_{p}^{0}(R)=\emptyset$, and $\sigma_{S F_{+}^{-}}(R \oplus S)=\sigma_{S F_{+}^{-}}(R) \cup \sigma_{S F_{+}^{-}}(S)$.

\section{References}

[1] P. Aiena and P. Peña, A variation on Weyl's theorem, J. Math. Anal. Appl. 324 (2006), no. $1,566-579$.

[2] M. Amouch and M. Berkani, On the property (gw), Mediterr. J. Math. 5 (2008), no. 3, $371-378$.

[3] M. Berkani, On a class of quasi-Fredholm operators, Integr. Equ. Oper. Theory 34 (1999), no. 2, 244-249.

[4] Index of B-Fredholm operators and generalization of a-Weyl's theorem, Proc. Amer. Math. Soc. 130 (2002), no. 6, 1717-1723.

[5] M. Berkani, N. Castro, and S. V. Djordjević, Single valued extension property and generalized Weyl's theorem, Math. Bohem. 131 (2006), no. 1, 29-38.

[6] M. Berkani and J. J. Koliha, Weyl type theorems for bounded linear operators, Acta Sci. Math. (Szeged) 69 (2003), no. 1-2, 359-376.

[7] M. Berkani and M. Sarih, On semi B-Fredholm operators, Glasg. Math. J. 43 (2001), no. 3, 457-465.

[8] M. Berkani and H. Zariouh, Extended Weyl type theorems, Math. Bohem. 134 (2009), no. $4,369-378$.

[9] S. Clary, Equality of spectra of quasisimilar hyponormal operators, Proc. Amer. Math. Soc. 53 (1975), no. 1, 88-90.

[10] L. A. Coburn, Weyl's theorem for nonnormal operators, Michigan Math. J. 13 (1966), $285-288$.

[11] S. V. Djordjević and Y. M. Han, A note on Weyl's theorem for operator matrices, Proc. Amer. Math. Soc. 131 (2003), no. 8, 2543-2547.

[12] B. P. Duggal and C. S. Kubrusly, Weyl's theorem for direct sums, Studia Sci. Math. Hungar. 44 (2007), no. 2, 275-290.

[13] A. Gupta and N. Kashyap, Generalized a-Weyl's theorem for direct sums, Mat. Vesnik 62 (2010), no. 4, 265-270.

[14] Y. M. Han and S. V. Djordjević, a-Weyl's theorem for operator matrices, Proc. Amer. Math. Soc. 130 (2002), no. 3, 715-722.

[15] R. E. Harte and W. Y. Lee, Another note on Weyl's theorem, Trans. Amer. Math. Soc. 349 (1997), no. 5, 2115-2124.

[16] H. Heuser, Functional Analysis, John Wiley \& Sons Inc, New York, 1982.

[17] K. B. Laursen and M. M. Neumann, An Introduction to Local Spectral Theory, Clarendon, Oxford, 2000. 
[18] D. C. Lay, Spectral analysis using ascent, descent, nullity and defect, Math. Ann. 184 (1970), 197-214.

[19] W. Y. Lee, Weyl spectra of operator matrices, Proc. Amer. Math. Soc. 129 (2001), no. $1,131-138$

[20] V. Rakočević, On a class of operators, Mat. Vesnik 37 (1985), no. 4, 423-426.

[21] _ Operators obeying a-Weyl's theorem, Rev. Roumaine Math. Pures Appl. 34 (1989), no. 10, 915-919.

[22] S. Roch and B. Silbermann, Continuity of generalized inverses in Banach algebras, Studia Math. 136 (1999), no. 3, 197-227.

\section{Mohammed Berkani}

DEPARTMENT OF MATHEMATICS

SCience Faculty OF Oujda

University MOHAMmed I

OpERATOR THEORY TEAM, SFO

OujDA, 60000, Morocco

E-mail address: berkanimo@aim.com

HASSAN ZARIOUH

DEPARTMENT OF MATHEMATiCS

Science Faculty of Meknes

University Moulay ISMaIL

Meknes, 50000, Morocco

E-mail address: h.zariouh@yahoo.fr 